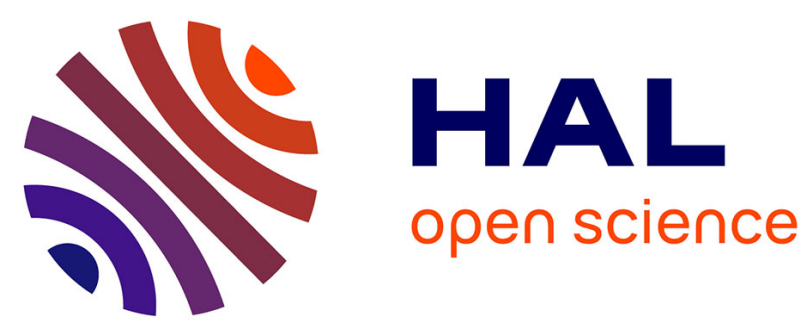

\title{
Software Defined Networking and Virtualization for Broadband Satellite Networks
}

\author{
Lionel Bertaux, Samir Medjiah, Pascal Berthou, Slim Abdellatif, Akram \\ Hakiri, Patrick Gelard, Fabrice Planchou, Marc Bruyère
}

\section{To cite this version:}

Lionel Bertaux, Samir Medjiah, Pascal Berthou, Slim Abdellatif, Akram Hakiri, et al.. Software Defined Networking and Virtualization for Broadband Satellite Networks. IEEE Communications Magazine, 2015, Satellite Communications and Networking: Emerging Techniques and New Applications, 53 (3), pp. 54-60. hal-01107652v2

\section{HAL Id: hal-01107652 \\ https://hal.science/hal-01107652v2}

Submitted on 21 Jan 2015

HAL is a multi-disciplinary open access archive for the deposit and dissemination of scientific research documents, whether they are published or not. The documents may come from teaching and research institutions in France or abroad, or from public or private research centers.
L'archive ouverte pluridisciplinaire HAL, est destinée au dépôt et à la diffusion de documents scientifiques de niveau recherche, publiés ou non, émanant des établissements d'enseignement et de recherche français ou étrangers, des laboratoires publics ou privés. 


\title{
Software Defined Networking and Virtualization for Broadband Satellite Networks
}

\author{
Lionel Bertaux ${ }^{\text {a,b }}$, Samir Medjiah ${ }^{\text {a,c }}$, Pascal Berthou ${ }^{\text {a,c }}$, Slim Abdellatif ${ }^{\text {d,a }}$, \\ Akram Hakiri $^{\mathrm{a}, \mathrm{b}}$, Patrick Gelard ${ }^{\mathrm{e}}$, Fabrice Planchou ${ }^{\mathrm{f}}$, Marc Bruyere ${ }^{\mathrm{a}, \mathrm{g}}$ \\ ${ }^{a}$ CNRS, LAAS, 7 avenue du colonel Roche, F-31400 \\ ${ }^{\mathrm{b}}$ Univ de Toulouse, LAAS, F-31400, Toulouse, France \\ ${ }^{\mathrm{c}}$ Univ de Toulouse, UPS, LAAS, F-31400, Toulouse, France \\ ${ }^{\mathrm{d}}$ Univ de Toulouse, INSA, LAAS, F-31400, Toulouse, France \\ ${ }^{\mathrm{e}}$ CNES, Centre National d'Etudes Spatiales 18 Av. Edouard Belin, 31400, Toulouse, France \\ ${ }^{\mathrm{f}}$ EADS Astrium, 31 rue des Cosmonautes, Z.I. du Palays, F-31402 Toulouse Cedex 4, France \\ ${ }^{\mathrm{g}}$ Dell Inc \\ \{firstname.lastname\}@\{laas|astrium|cnes $\}. f r$
}

"(c) 2015 IEEE. Personal use of this material is permitted. Permission from IEEE must be obtained for all other users, including reprinting/ republishing this material for advertising or promotional purposes, creating new collective works for resale or redistribution to servers or lists, or reuse of any copyrighted components of this work in other works." 


\title{
Software Defined Networking and Virtualization for Broadband Satellite Networks
}

\author{
Lionel Bertaux, Samir Medjiah, Pascal Berthou, Slim Abdellatif, Akram Hakiri, Patrick Gelard, Fabrice Planchou, and Marc \\ Bruyere
}

\begin{abstract}
Satellite networks have traditionally been considered for specific purposes. Recently, new satellite technologies have been pushed to the market enabling highperformance satellite access networks. On the other hand, network architectures are taking advantages from emerging technologies such as Software-Defined Networking (SDN), network virtualization and Network Functions Virtualization (NFV). Therefore, benefiting communications services over satellite networks from these new technologies at first, and their seamless integration with terrestrial networks at second, are of great interest and importance. In this paper, and through comprehensive use cases, the advantages of introducing network programmability and virtualization using SDN and/or NFV in satellite networks are investigated. The requirements to be fulfilled in each use case are also discussed.
\end{abstract}

Keywords-Broadband Satellite networks, Software Defined Networking, Network virtualization, Network Function Virtualization, OpenFlow

\section{INTRODUCTION}

Software-defined networking (SDN) is a new paradigm shift in communication networks, receiving an increasing attention from industry and academia. Similarly, network virtualization and Network Functions Virtualization (NFV) are gaining adhesion in the telecom industry. Coupled together, they pave the way to new opportunities in network design, operation and management.

Terrestrial and satellite networks have always evolved differently. On one hand, terrestrial networks are in constant evolution and are already moving to embrace SDN and NFV [1]. On the other hand, satellite networks have traditionally been considered for specific purposes or as a backup technology (in established markets such as air and sea coverage) and thus rely on technologies locked down by major actors.

Network programmability, openness and virtualization are the key words of today networking architectures. Adopting these principles in satellite communications can help reducing CAPEX and OPEX, enhancing the performance and the QoS delivered to satellite communication end-users, extending the range of applications of satellite communications, and achieving seamless integration with terrestrial networks.

In this paper, we investigate how SDN, network virtualization and NFV can enhance satellite architecture to achieve the aforementioned objectives. Through practical usecases, we demonstrate benefits resulting from the integration of these emerging paradigms into communication satellite networks. We also highlight necessary requirements that have to be fulfilled.

This paper is organized as follows: background knowledge on software-defined networking, network virtualization and network function virtualization is presented in Section II. Section III gives a brief overview of the satellite network architecture considered in this study. Section IV details use-cases where SDN, network virtualization and NFV can be beneficial to satellite networks. Finally, Section V concludes on their global contributions to communication satellite networks.

\section{BACKGROUND}

\section{A. Introduction to Software-Defined Networks}

Recently, SDN [2] has emerged as a new approach for network programmability and management, where the centralized control plane logic is decoupled from the dataforwarding plane. SDN architectures define a new entity (called controller) that centralizes control intelligence of one or more network elements (basically switches) as shown in Figure 1. Various open interfaces [3] have been defined to communicate between control plane and data plane (South Bound interface), and OpenFlow [4] is a de-facto standard. On the North interface of the controller, applications can be deployed with a network-wide view of data path elements.

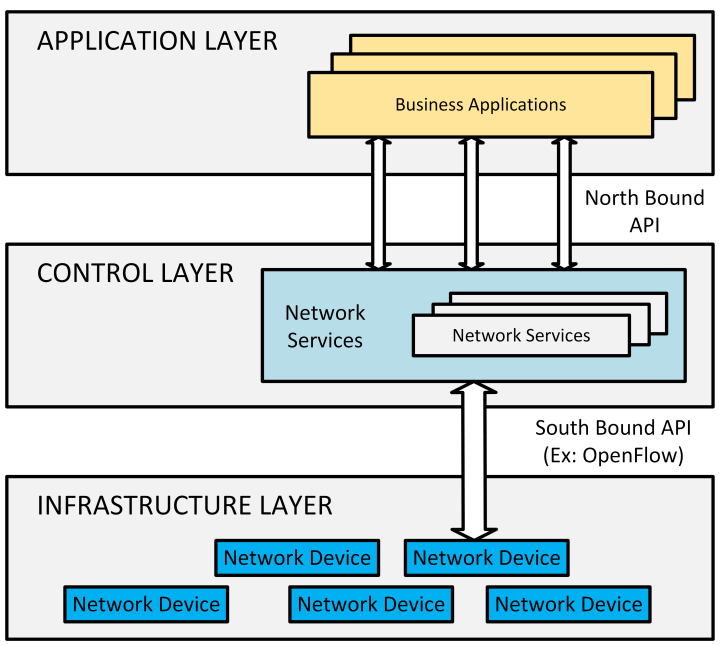

Figure 1 - Network vision with the Software-Defined Network (SDN) approach.

SDN opens up new opportunities. Most notably, it simplifies network management [5] and allows automated 
customized on-demand networking with optimal network resource utilization.

\section{B. Network Virtualization}

Network virtualization enables the creation and coexistence of multiple isolated and independent virtual networks over a shared network infrastructure [6]. A virtual network is a logical network with some of its elements (network devices (or nodes) and links) virtual. A virtual node is an abstraction of a network device that is often hosted on a single physical node. It executes network functions such as routing, forwarding, etc. by consuming part of the resources of the hosting node. The resources allocated to a virtual network device are as diverse as CPU, Volatile Memory, network interfaces, storage, switching, etc. Similarly, a virtual link is an abstraction of a network link that is established on one or multiple physical links or physical paths. It consumes transmission resources (i.e. physical links' bandwidth) as well as switching resources at the traversed physical nodes.

\section{Network Function Virtualization}

Historically, the telecom industry has always privileged the use of dedicated equipment to provide network functions. However, this model inevitably leads to long time to market delays and important costs. This model is being questioned by the NFV concept [7]. Indeed, NFV advocates the virtualization of network functions as software modules running on standardized IT infrastructure (like commercial off-the-shelf servers), which can be assembled and/or chained to create services. This approach makes use of the experience on server virtualization got from the cloud computing industry since a virtual network function may be implemented on one or more virtual machines.

The main benefits of NFV are reduction of CAPEX and OPEX and improved network agility.

\section{SATELlite NeTWORK ARCHITECTURE}

This work considers a typical Broadband Satellite Network (BSN) that provides a multi-beam coverage with forward and return links. The ground segment of the BSN gathers multiple Hubs that are interconnected via a dedicated backbone network with some PoPs (Point of Presence) or gateways to external networks, typically the Internet (see Figure 2).

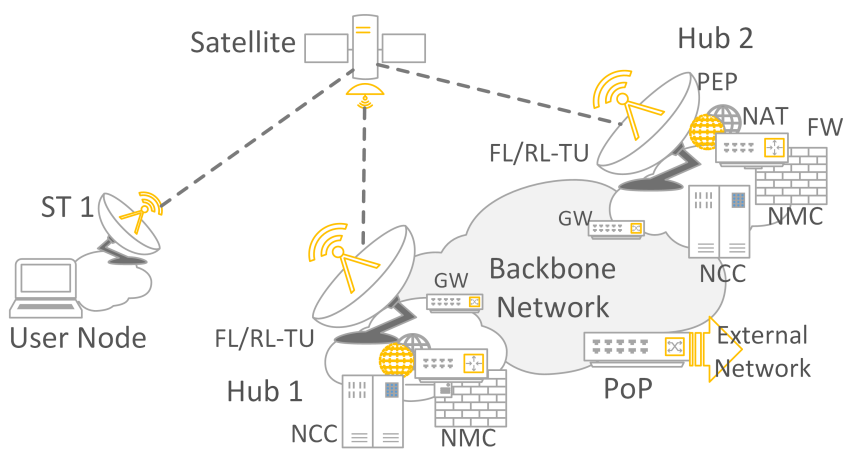

Figure 2 - Satellite Communication Architecture.
Generally, a Hub supports bidirectional traffic on one or many beams. It combines a Forward Link Transmission Unit (FL-TU) and a Return Link Reception Unit (RL-RU) with a Gateway (GW) to terrestrial networks, a Network Control Centre (NCC) and a Network Management Centre (NMC). The FL-TU performs baseband related functions like DVB-S2 coding and modulation with Adaptive coding and modulation (ACM). The Gateway is typically a full-featured IP router with a strong set of functions and protocols (e.g. support for various routing protocols, Network Address Translation, Access Control Lists (ACLs) and firewall services, SNMP, QoS, etc.). The NCC provides control functions; it typically performs Satellite Terminals (ST) admission control and resources control/allocation on the forward and return links. The NMC performs all management functions, i.e. network element's (ST, Hub) configuration, as well as fault, performance, accounting and security management. Performance Enhancing Proxy (PEP) designed to improve TCP performance over satellite links may also be co-located at the Hub (or deported at the PoPs or closer to end-users).

The successful delivery of satellite communication services to end-users involves one or many real-life business actors, each playing one or many roles (with the set of functional responsibilities they assume). Referring to [8], three major roles are distinguished:

- Satellite Operator (SO): it owns the satellite and assumes its operation. It leases satellite capacity at the transponder level (physical layer) to one or several SNOs.

- Satellite Network Operator (SNO): it operates a broadband satellite network with one or more satellite transponders and one or more satellite hubs. It provides satellite forward and return links to second-tier operators by dividing transponder level bandwidth. The NCC controls this bandwidth sharing. Via the NMC, the SNO provides a management interface to the purchased resources.

- Satellite Virtual Network Operator (SVNO): Based on the satellite links contracted from one or multiple SNOs, it builds and provides end-to-end higher-level added-value services that are made available via a satellite access.

\section{USES CASES}

\section{A. Use case 1 : Inter-hub handover with Site Diversity}

\section{1) Description and current practices}

In satellite communications, the use of high frequency bands such as $\mathrm{Ka}$ or $\mathrm{Q} / \mathrm{V}$ makes Adaptive Coding and Modulation (ACM) mechanisms mandatory to counteract signal degradation due to meteorological events like clouds or rain. In case attenuation is extreme, throughput reduction due to ACM and induced network congestion may not be in agreement with QoS constraints of some flows (VoIP, video conference). If meteorological degradations are due to weather disturbances over ST localization, using robust coding 
mechanisms cannot be avoided. If degradations concern Hub localization, using another distant site should be considered. The concept of connecting (successively or not) a single ST to several Hubs is called site diversity.

Site diversity deployment can follow two different approaches as described in [9]. The $\mathrm{N}+\mathrm{P}$ approach relies on $\mathrm{P}$ redundant Hubs that can replace failing sites, resulting in a full handover (HO) of users. The $\mathrm{N}+0$ approach uses frequency multiplexing to serve ST with carriers from different Hubs, a failing site inducing losing the corresponding portion of frequency band. Both cases raise some challenges if network service continuity with performance impairments must be guaranteed. Indeed, a hub change (case $\mathrm{N}+\mathrm{P})$ or a carrier modification $(\mathrm{N}+0)$ has to be signaled to STs and executed. Simultaneously, routing tables have to be updated in the terrestrial network. Also, the handover decision problem is complex since it may concern hundreds of STs and account for several criteria such as flow observation, network knowledge, changing channels quality, etc. Current satellite networks follow the $\mathrm{N}+\mathrm{P}$ approach with handover performed for a whole beam at a time.

\section{2) SDN opportunities for site diversity}

Obviously, applying SDN principles in the context of site diversity can help devising an effective handover decision algorithm as well as easing the execution of the handover. Basically, this can be achieved with the following enhancements shown in figure 3 :

- SDN-enabled switches to replace GWs in Hubs,

- One SDN (OpenFlow) master controller located in a hub site running the network application in charge of inter-hub handover management. For clarity reasons, one SDN controller is depicted in Figure 3, however the use of several controller entities should be considered for scalability and reliability purposes.

- Interfaces to NCC and NMC exposed to the handover application that gather monitoring information and allow triggering some ST configurations

- Optional: a SDN enabled backbone network

The handover management application decides when a handover is needed (and the flows or STs that are concerned by the handover) based on:

- Flow constraints: QoS requirements, specific user SLA,

- Flow monitoring: to identify active services, the performance and resources (satellite and backbone) that they are receiving;

- Satellite network as well as terrestrial backbone network performance indicators

- SNO/SVNO policy: emergency cases or super-user demands,

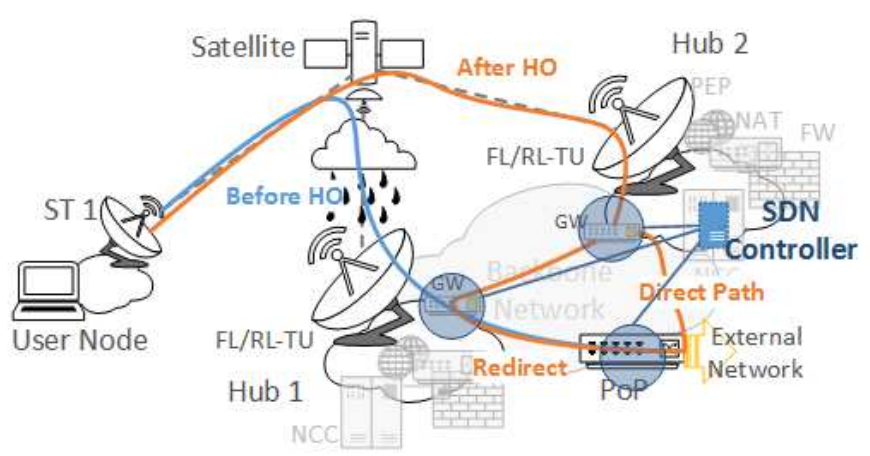

Figure 3 - SDN architecture of terrestrial network in site diversity context

Once handover of certain flows or STs is decided, the application automatically:

- Informs concerned ST and FL/RL-TU to change their frequencies if needed,

- Updates forwarding rules in the GWs and backbone network.

Two options can be considered:

- "Direct Path Routing": Flows are routed directly from their new hub to the nearest PoP.

- "Traffic Redirection": Flows are still passing through their home hub site after having been redirected, at their new hub, via the backbone network,

This is achieved thanks to SDN related programmable functionalities that are added to the packet-processing pipe. For example, OpenFlow can dynamically deploy forwarding rules matching packets based on:

- Incoming network interface,

- IP/MAC addresses,

- Classes of services or protocols used,

- Rate of identified flow or group,

- Deep Packet Inspection (DPI) using legacy functions.

In conclusion, SDN doubtless contributes to present and future satellite networks by easing the management of interhub handover enabled by site diversity, but also, by extending its capabilities.

\section{B. Use case 2 : Enhancing $V N O$ services}

\section{1) Description and state of the art}

The need for Virtual Network Operator (VNO) services is clear and not new. VNO services allow SNOs to partition their satellite resources between multiple SVNOs efficiently by delivering dedicated satellite capacities with different levels of QoS guarantees. Typically, SVNOs in turn repackage these services to provide their customers with added-value end-to-end services. This business model has been for a while in the satellite market landscape. However, the level of control that SVNOs have on their purchased services (and underlying resources) is limited mainly because of the closed nature of 
satellite devices and the management interface between SNOs and SVNOs.

Figure 4 presents on the one hand, the Network Management System (NMS) used by a SVNO, and on the other hand the SNO's NMC that manages the NCC, GW as well as all STs. Even if some management functionalities can be done directly from SVNO's NMS to its STs (e.g. routing, etc.), most must go through the NMC (e.g. to get STs' status and statistics). To this end, a management interface (I.SNOSVNO) is provided by the SNO as part of the VNO service to let the NMS manage SVNO's satellite terminals. This interface is usually SNMP based complemented with some vendor specific solutions.

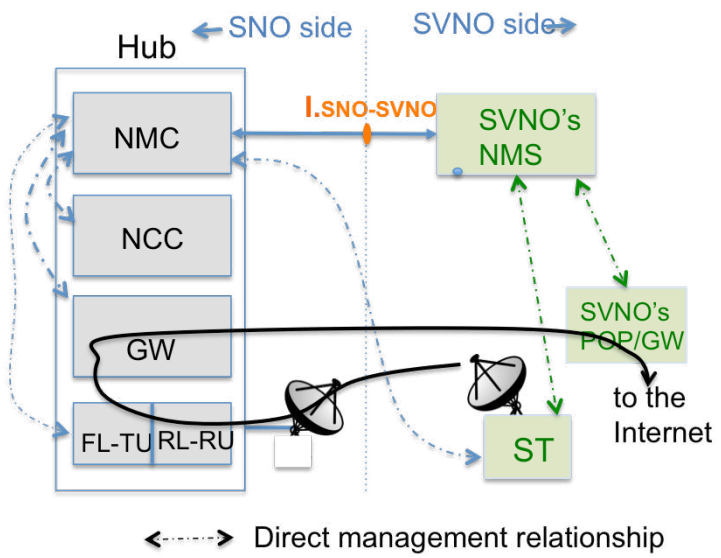

Figure 4 - SNO and SVNO Management relationships

The VNO services provided to SVNOs are dependent on the level of visibility and control capabilities exposed by the I.SNO-SVNO management interface that is far from being comprehensive. Moreover, some control capabilities require human intervention from the SNO to validate or perform the required configuration. From the SVNOs perspective, this refrains the development of novel services and complicates the provision process of the services they offer.

\section{2) Network virtualization and network programmability opportunities for VNO services}

SVNOs are asking for more control on their resources with reduced (if no) intervention from the SNOs. The issues are (1) quicker automated service provisioning processes, (2) enriching their service catalogue, and (3) enabling Satellite Communication as a Service consumption model.

Opening satellite devices via programmatic interfaces (with a rich set of instructions that goes beyond SNMP capabilities) exposed to second tier operators coupled with network virtualization is the way to follow in order to fulfill these goals. By applying device virtualization (i.e. server virtualization applied and adapted to network devices) to SNO satellite hubs, a virtual Hub can be assigned on a per SVNO basis (see Figure 5). With the guarantees brought by isolation - which is a key feature of network virtualization, and applies to data, control and management planes as well as to performance and security) - a SNO can delegate the full control and management of virtual hubs to their customer SVNOs. Therefore, SVNOs can independently enforce their own policies on their satellite virtual networks. Having the control on the NMC to NMS management interface (the range of its capabilities), SVNOs can make the provisioning process of the services delivered to their customers fully automated. Indeed, a provisioning engine can be used to orchestrate and perform all the required configurations by accessing the above-cited interface. Moreover, dynamic SLA can be easily supported. Indeed, a SVNO subscriber may ask via a secured portal to dynamically change his bandwidth requirement, the provisioning engine can then autonomously take the right configuration actions to provide and enforce the newly requested SLA within a time frame of few minutes (in fact, such service exists but with higher response times, often with human in the loop). New services, such as you pay what you use services can also be considered.

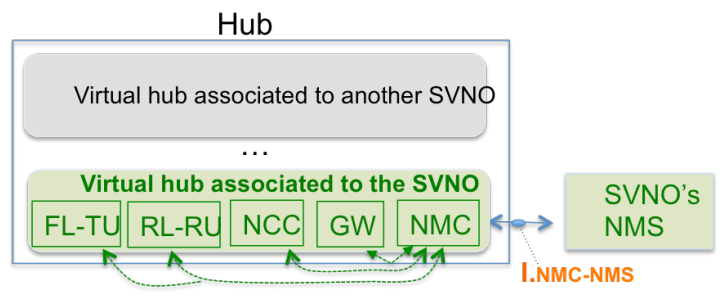

Figure 5 - Hub virtualization

A further step can be achieved by introducing programmability thus enabling a programmable virtual hub assigned to SVNOs. Programmability may concern the control plane (routing, forwarding, monitoring as stated in SDN) allowing SVNOs to devise their own customized traffic control schemes but also the data plane allowing SVNOs to devise customized packet processing algorithms (e.g. PEP, encryption). It paves the way for the diversification and enrichment of the services that SVNOs provide.

The road to programmable virtual satellite networks is just in its beginning. Network device virtualization with the stringent level of isolation that is required by satellite operators needs to be defined in order to extend the scope of the SVNO to SNO management interface (i.e. I.NMCNMS). Some vendors already provide some form of Hub virtualization; it mainly concerns the gateway (GW) and the management center (NMC) with dedicated I.NMC-NMS to SVNOs. Extending the application of some form of virtualization to the other hub's elements is technically tractable within a reasonable time frame. But, devising a Hub virtualization technique that allows a comprehensive management interface between SNOs and SVNOs is still a research topic. Similarly, programming capabilities need to be identified and defined both at the control plane where SDN/OpenFlow capabilities need to be extended or completed with accompanying protocols, and possibly, at the data plane. This is another research topic with outstanding issues. 


\section{Use case 3 : Satellite and Terrestrial Networks Integration}

\section{1) Description and state of the art}

Whether to provide data backhauling (mobile, military, marine, etc.), or to efficiently deliver communications services, along terrestrial access networks, in some deployments environments known as "gray areas" (areas with a limited internet connectivity i.e. $<512 \mathrm{Kbit} / \mathrm{s}$ ), hybridation of different access networks with satellite would help providing an efficient service offer. Some of its advantages include:

- Capacity aggregation: some applications may require more bandwidth than what a single link provides. In this case, multi-link transmission will help achieving the total required bandwidth. To improve service quality, the additional links may be used to serve a specific purpose e.g. error correction data.

- Load balancing: Data flows from different applications may be forwarded through different links in order to keep the link utilization at its lower level. Similarly, in order to enhance service functionality, the choice of link can be application driven.

To make such solution a reality, the system architecture should provide a fine-grained control over the carried data flows. Indeed, the ability to dispatch any data stream or any portion of it over the best link is essential. This routing should be done in a seamless fashion to the deployed applications. Nowadays, such control can be sought through a complex combination of various techniques such as Policy-Based Routing (PBR), multi-link protocols (MLPPP, SCTP, etc.), and traffic identification mechanisms (Packet marking, DPI, Layer-7 filters, etc.). However, it is worth to notice that all these techniques fail to provide the necessary control level for data flows dispatching over different links. Moreover, their behavior lacks dynamicity since forwarding rules are static and do not take into account the evolving link conditions and applications flows.

\section{2) SDN-Enabled Satellite/ADSL Integration}

SDN paradigm can play an important role in such solution. Indeed, SDN-based implementation of the hybrid architecture can bring the appropriate control level that current protocols and mechanisms cannot efficiently achieve. Moreover, since packets forwarding decisions are made upon matching rules on packets headers, convergence between the different involved networks, running different communication technologies, can be either achieved at level 3 or at a lower level (level 2).

Figure 6 gives an overview of a network architecture that makes use of both an ADSL access network and a bidirectional satellite network. In this architecture, the global Network Provider (NP) operates both access networks.

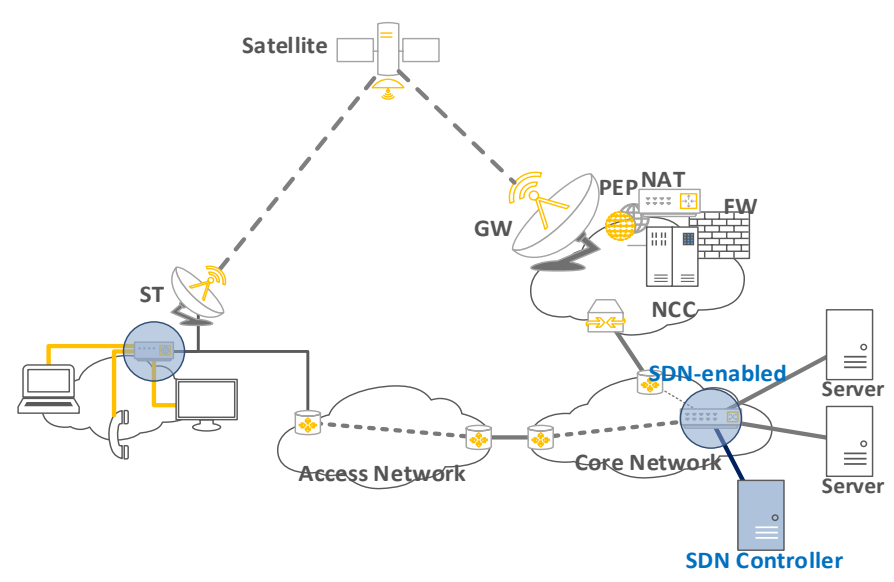

Figure 6 - SDN-Enabled Satellite/ADSL Hybrid Architecture

In this scenario, the network operator deploys SDNenabled devices within its network infrastructure, but also at the customer/user premises. Indeed, in this case, the home gateway becomes SDN-enabled and operates under the supervision of an SDN Controller hosted at the network operator. Thanks to a network application running on top of the SDN controller, data flows dispatching can be achieved at either the forward or the return links.

In the context of triple-play services over hybrid Satellite/ADSL architecture, the freedom in packet forwarding brought by SDN (i.e. OpenFlow packet forwarding rules) enables various scenarios. For example, when starting a phone call and in order to meet the QoS requirement of VoIP, lowlatency link (e.g. ADSL) can be temporarily and dynamically reserved to voice packets while all other data packets being transmitted over this link are redirected to the satellite link.

However, an SDN-based solution has the following requirements:

- Data flow identification: for efficient flow dispatching, the control application needs to identify the services data flows based on parameters such as: IP addresses, port numbers, TOS, or any byte pattern in packets headers or payloads. Thanks to OpenFlow rules expression, such patterns can be easily implemented.

- Link monitoring: the control application needs to constantly monitor the links in terms of latency, available bandwidth, etc. in order to optimize the data flows dispatching. OpenFlow in its version 1.3 introduces metering tables, a powerful tool to gather per switch port or even per data flow statistics.

- Dynamic forwarding rules generation and update: the control application needs to react to any changes in the links conditions and generate/deploy the appropriate forwarding rules or update the already established ones.

Finally, SDN can make hybrid architecture more efficient and ease its deployment. Moreover, it will enable novel and innovative services and applications. This use case is already a reality since SDN-enabled switches (e.g. 
OpenFlow compatible switches) are already on the market, however the hybridization applications and strategies have to be developed. SDN enabled hybrid and integrated setup boxes must also be proposed.

\section{Use case 4 : middleboxes virtualization}

Middleboxes are prevalent in the Internet architecture, and especially within specific networks such as satellite communication networks. These smart entities are used for various purposes as performance optimization, security, and address translation. This section analyzes how NFV can improve the classical PEP functions in satellite networks.

\section{1) TCP performance optimization.}

The TCP/IP model was shown to be not optimum, in terms of performance, in certain WAN and particularly in constrained environments, such as satellite networks. Various TCP protocol versions targeting satellite networks were proposed with the objective of improving the performance of TCP. They were, however, confronted with issues related to their deployment on user terminals. The solution that was found and which is still in use today is to insert devices, at the boundaries of the satellite network, to transform the operation of TCP into a satellite compatible version. These devices, called Performance Enhancing Proxy (PEP) [10], were spread in the satellite networks, offering by the same occasion advanced services such as Web Caching.

Protocol optimizations provided by PEPs are not compatible with several scenarios, in particular, in military or aeronautical contexts where security and mobility constraints are present. For instance, implementing a mobile architecture, such as mobile IP, poses complex problems to solve for PEPs. The most problematic case happens during a hybrid handover, i.e. from a satellite network requiring PEP optimization to a network where it is no longer necessary (and potentially counter-productive). In this scenario, TCP connections managed and accelerated by a PEP should survive to a deactivation of the PEP (or more generally to a change of PEP). However, PEPs are physically locked to the infrastructure and cannot move to follow the end-user. Solutions to this problem are proposed in [11] for hybrid satellite/terrestrial; they require context exchanges between PEPs.

Other middleboxes that provide advanced services in satellite networks (NAT, firewall, security, etc.) are also subject to the same issues.

\section{2) PEPs and Network Function Virtualization}

The Network Function Virtualization paradigm aims at implementing data-plane processing or control-plane functions in high volume data centers or network elements. This open a new era in thinking middleboxes, as they could be easily deployed, on demand, and under the control of an operator to provide advanced services. Moreover, these middleboxes could be mobile, as it is only relying on software that can be migrated from a standard server to another.
Considering use case \#1 presented above (on site diversity), PEPs are typically implemented in the satellite hubs. When a Satellite Terminal hands over to a new hub, its TCP connections that cross the PEP will be broken as the new PEP will not be aware of connections' contexts.

With the NFV paradigm, PEPs will no longer be implemented as a dedicated middlebox but rather in software that can be run on different devices. Moreover, the PEP function can be dedicated to a communication context (dedicated to an ST for instance) and can be tuned according to the application requirements (security, mobility, performances, etc.) If an ST makes a handover from one satellite hub to another one, its "dedicated virtual PEP" will migrate to the new hub and will continue to perform the appropriate TCP optimization.

Several cloud computing platforms support NFV and already offer a solution to deploy Virtual Network Functions (VNF). Virtual functions implementing TCP optimization and acceleration for web application servers are proposed by some vendors. From a technical perspective, PEP virtualization can shortly become a reality.

\section{CONCLUSION}

Through the description of four real world use cases, this paper has shown some opportunities brought by the SDN and NFV paradigms to broadband satellite networks as well as their impacts on typical satellite system architecture. SDN and NFV are complementary solutions. SDN brings flexibility, automation and customization to the network. NFV brings agility in the delivery of services and reduces time-to-market development of new services. There is no doubt that they will take a central place in future satellite communication systems.

\section{ACKNOWLEDGEMENTS}

This work is partially funded by the French National Research Agency (ANR), the French Defense Agency (DGA) under the project ANR DGA ADN (ANR-13-ASTR-0024) and the French Space Agency (CNES).

\section{REFERENCES}

[1] Open Networking Foundation, Use Cases and Migration Methods, Migration Working Group, ONF white paper, 2013.

[2] Open Networking Foundation, "SDN Architecture Overview", December 2013.

[3] B. Astuto A. Nunes et al,, A Survey of Sofware-Defined Networking: Past, Present, and Future of Programmable Networks, IEEE Communications Surveys \& Tutorials 2014.

[4] Open Networking Foundation, "OpenFlow Switch Specification version 1.4.0 (Wire Protocol 0x05)", Oct. 2013.

[5] K. Hyojoon and N. Feamster, "Improving network management with software defined networking", IEEE Communications Magazine, vol. 51, no. 2, pp 114-119, Feb. 2013.

[6] N. M. Mosharaf Kabir Chowdhury and Raouf Boutaba. Network virtualization: state of the art and research challenges. IEEE Communications Magazine, vol. 47, no.7, pp 20-26, July 2009

[7] ETSI GS NFV 001 V1.1.1, "Network Functions Virtualisation (NFV) : Use Cases", Oct. 2013.

[8] ETSI TS 101 545-1 V1.1.1, "Digital Video Broadcasting (DVB); Second Generation DVB Interactive Satellite System (DVB-RCS2); Part 1: Overview and System Level specification”, May 2012. 
[9] N. Jeannin, L. Castanet, J. Radzik, M. Bousquet, B. Evans, P. Thompson, "Smart gateways for terabit/s satellite.", Int. J. Satell. Commun. Network., 32: 93-106, Jan. 2014.

[10] J. Border and al, "Performance Enhancing Proxies Intended to Mitigate Link-Related Degradations", IETF RFC 3135, Jun. 2001.

[11] E. Dubois, J. Fasson, C. Donny, E. Chaput, "Enhancing TCP based communications in mobile satellite scenarios: TCP PEPs issues and solutions“, ASMS 2010, Cagliari, Italy, p 476- 483, Sep. 2010.

\section{BIOGRAPHIES}

Lionel Bertaux received a $\mathrm{PhD}$ (2013) in computer science from the University of Toulouse, France. He is a postdoctoral researcher at the Laboratory for Analysis and Architecture of Systems of the French National Centre for Scientific Research (LAAS-CNRS).

Samir Medjiah received a $\mathrm{PhD}$ (2012) in computer science from the University of Bordeaux, France. He is Associate Professor at Paul Sabatier University in Toulouse (France) and a research scientist at the Laboratory for Analysis and Architecture of Systems (LAASCNRS). His main research interests include overlay networks optimization, network virtualization, and software defined networking. He has worked on various $R \& D$ projects related to terrestrial/satellite networks convergence, application-driven networking, and Network-Application co-optimization.

Pascal Berthou received a $\mathrm{PhD}$ in computer science and telecommunication from national polytechnic institute of Toulouse in 2001. He is associate professor at Université Paul Sabatier. He joins the Laboratory for Analysis and Architecture of Systems of the French National Centre for Scientific Research (LAAS-CNRS) in 1998 as a research staff member, where he works in the area of highspeed networks and protocols and multimedia communications. Since this date he has covered two major areas of activity. The first area deals with Satellite communication systems. The second research area is sensor networks, particularly the communication system and their application, which in recent years has been directed towards the design of WSN for instrumentation networks. Within this domain he has focused on hardware/software network interface issues and cross layering interactions to reduce the energy consumption in WSN. He recently started having a research contribution in the area of SDN.

Slim Abdellatif received the M.S. (1998) and the Ph.D. (2002) degrees in computer science both from the University of Toulouse, France. He is currently an Assistant Professor at the Institut National des Sciences Appliquées of Toulouse and a research scientist at the Laboratoire d'Analyse et d'Architecture des Systèmes (LAAS) from the French National Center for Scientific Research (CNRS). His current research interests include network virtualization, Software Defined Networking, QoS and resource management in wireless networks.

Akram Hakiri is an Associate Professor of Electrical Engineering and Computer Science and research scientist at ISSAT Mateur, Tunisia. His current research focuses on developing novel solutions to emerging challenges in wireless mobile networks, Cloud computing, SDN, and QoS.

Patrick Gelard is a network \& telecom research engineer, senior expert at Centre National d'Etudes Spatiales (CNES), Toulouse, France. Began his professional experience at ABS (Alcatel Bussiness
System) on PABX during 4 years before joining the CNES in 1990 as protocol engineering expert at information systems division. For over eight years now, he works in CNES at Network and telecommunication unit as a terrestrial and satellite networking expert. The main projects in telecommunications In which he was involved at CNES are:

- Development of an innovative "IP-Phone" system design working on Ethernet Lan.;

- Work on dedicated ATM infrastructure design for synchronous $50 \mathrm{Mb} / \mathrm{s}$ spacecraft telemetry real time delivery (named ATLAS),

- Develop a tool of remote teaching and E-Learning base on "Java Multimedia Framework" for hybrid context Broadcast satellite/broadband Terrestrial,

- $\quad$ Design of an operational "heavy duty" (300 Gbytes/day) remote archiving system,

- Design of a ground communication system for satellite telemetry monitoring and automated MIT alert (HETE2 Project),

- $\quad$ Participated at satellite E-learning experiment over 250 French schools. Internet proxying, caching, streaming, multicast and asymetric routing architectures,

- Development of an open multimedia DVB-S experimental platform,

- Expertise in satellite communications project at CNES (Agora, ATF, Athena-Fidus, MM2G, THD, SMILE).

It has now been five years that he has in charge of research axis "terrestrial and satellite infrastructure convergence". This led him to work on "Software Defined Networking and Virtualization for Broadband Satellite Networks".

$\mathrm{He}$ is going to work in Vital H2020 project (VIrtualized hybrid satellite-TerrestriAl systems for resilient and fLexible future networks) as network expert. VITAL is an ambitious proposal addressing the combination of terrestrial and satellite networks by pursuingtwo key innovation areas, namely (1) bringing Network Functions Virtualization (NFV) into the satellite domain and (2) enabling Software-Defined-Networking (SDN)based, federated resources management in hybrid satelliteterrestrial networks.

Fabrice Planchou graduated from ENSEEIHT (Toulouse, 1990), belongs to Airbus Defence \& Space (ex-Astrium) since 1990, dealing with telecom space systems. He heads an engineering team devoted to $R \& T$ and satellite telecom system validation. His main interest is the promotion of space systems within the telecom service market. Main researches and developments are based around terrestrial/satellite hybrid systems, mobiles systems, for both broadband and broadcast services, and also the M2M domain.

Marc Bruyere Marc Bruyere is a Technical Consultant at DellForce 10 and a Phd student at the LAAS /CNRS at Toulouse. In 1996 he worked for the ISP Club-Internet.fr. He worked for Cisco, Vivendi Universal, Credit Suisse First Boston, Airbus/Dimension Data, Force10 Networks and Dell. He is a Cisco Certified Internetwork Expert \#16651. He is involved in the NetFPGA project for few years and his thesis is about a new architecture for IXP OpenFlow/SDN environment. 